\title{
Profesores Noveles y Representaciones de Género en el Marco de la Educación Inclusiva
}

\section{Novice Teachers and Gender Representations in the Framework of Inclusive Education}

\author{
Violeta Quiroz-Fuentes ${ }^{1 *}$ \\ Melissa Sennas-Vásquez ${ }^{2}$ \\ Sylvia Contreras-Salinas ${ }^{3}$ \\ ${ }^{1}$ The Little School, Chile \\ ${ }^{2}$ Cristóbal Colón, Melipilla, Chile \\ ${ }^{3}$ Universidad de Santiago de Chile
}

\begin{abstract}
Aportando a la discusión que cuestiona la educación sexista y su permanencia dentro del sistema escolar chileno se decide desarrollar un estudio, el cual tiene como principal objetivo describir las representaciones de género que poseen las y los profesores noveles de educación general básica, cuyo marco de desarrollo profesional es la Educación Inclusiva. Se enmarcó en un enfoque cualitativo descriptivo, utilizando como herramienta de recolección de información entrevistas semiestructuradas a una muestra intencionada de participantes. Entre los hallazgos se destaca que en los discursos de los participantes se aprecia una transición en la categoría de género asociado al biologismo y también a lo sociocultural, observándose además inconsistencias y contradicciones en sus discursos respecto a la Educación Inclusiva. Así mismo, las conclusiones resaltan las propuestas que señalan los participantes para los programas de Formación Inicial Docente en el marco de una Educación Inclusiva, siendo la afectividad y performatividad del lenguaje aquellos elementos que inciden en el posterior desarrollo profesional.
\end{abstract}

Descriptores: Rol sexual; Docentes; Educación inclusiva; Formación de docentes; Chile.

Contributing to the discussion that questions sexist education and its permanence within the Chilean school system, it is decided to develop a study, which has as main objective to describe the gender representations of the novice teachers of basic general education, whose development framework Professional is Inclusive Education. It was framed in a descriptive qualitative approach, using semi-structured interviews as an information collection tool for an intentional sample of participants. Among the findings, it is highlighted that in the participants' speeches there is a transition in the gender category associated with biologic and also the sociocultural, while observing inconsistencies and contradictions in their speeches regarding Inclusive Education. Likewise, the conclusions highlight the proposals that participants indicate for the Initial Teacher Training programs within the framework of Inclusive Education, being the affectivity and performativity of the language those elements that affect the subsequent professional development.

Keywords: Gender roles; Teachers; Inclusive education; Teacher education; Chile.

*Contacto: quiroz.violeta@gmail.com

ISSN: 0718-7378

www.rinace.net/rlei/
Recibido: $\quad 15 / 07 / 2019$

$1^{\text {a }}$ Evaluación: 15/09/2019

Aceptado: $\quad 01 / 10 / 2019$ 


\section{Introducción ${ }^{1}$}

En los últimos años a nivel mundial, hemos sido testigos de significativos procesos de cambio: políticos, científicos, financieros, educativos, entre otros; dirigidos al pleno reconocimiento de los derechos humanos. En este escenario de carácter global, enmarcado entre la dualidad de las ofensivas de las demandas sociales y las respectivas respuestas gubernamentales, las autoridades chilenas han buscado solucionar tal dicotomía por medio de la promulgación de normativas afines a la movilización social.

Como ejemplo de lo anterior, destacan en la última década la Ley Antidiscriminación (Ley 20.609), la Ley de Inclusión Laboral (Ley 21.015) o la Ley de Identidad de Género (Ley 26.743) orientadas a velar y reconocer a otros como miembros de una sociedad en igualdad de derechos y oportunidades, con finalidad de desplazar toda acción que involucre una discriminación arbitraria. Particularmente, en materia educativa se creó la Ley General de Educación, la cual establece como deber del Estado velar por la igualdad de oportunidades e inclusión educativa, reduciendo las desigualdades derivadas de circunstancias económicas, sociales, étnicas, de género o territoriales (L.G.E., 2009, art. 4). Asimismo, se creó la Ley de Inclusión Escolar (Ley 20.845), creando nuevos criterios de trabajo en las escuelas, eliminando barreras de acceso y permanencia en el sistema educativo.

En materia de género, para asegurar estos procesos normativos, es posible identificar guías como el Plan de Educación para la Igualdad de Género (2015-2018), las Orientaciones para un uso de lenguaje no sexista e inclusivo (2017) y el Ordinario 768: Derecho de niñas, niños y estudiantes trans en el ámbito de la educación (2017); documentos que probablemente ponen en evidencia la necesidad y voluntad en enmarcar los proyectos educativos que deben avanzar hacia una educación no sexista.

Sin embargo, a pesar del avance legislativo señalado anteriormente, no podemos ignorar la creciente movilización feminista secundaria y universitaria en Chile del año 2018, la cual perturba la agenda nacional. Jóvenes de instituciones públicas y privadas paralizan sus estudios solicitando cambios radicales en el sistema político, económico, social y educativo, históricamente reconocido como masculino y hegemónico (Bonino, 2003; Cabello, 2018; Ceplak, 2013; Strear, 2017). Una de las peticiones centrales que exige este movimiento es la construcción de políticas con perspectiva de género que cercenen el enfoque patriarcal de la educación sexista, su lenguaje discriminatorio y la reproducción de los roles de género, etcétera (Zerán, 2018). En este escenario, la escuela, en su rol de aparato ideológico del Estado (Althusser, 1988), es una incubadora por excelencia de estereotipos que contribuyen a generar desigualdades en términos de género (Flores, 2007). Considerando esto, las escuelas y sus actores legitiman discursos que asumen que la diferencia es una condición natural de los individuos, dejando a un lado la reflexión respecto a las formas de razonamiento como, por ejemplo, las creencias, estereotipos, criterios de categorización, entre otras, que crean esas diferencias.

En este sentido, existen discursos históricamente validados que conllevan a prácticas y criterios de categorización en las y los estudiantes, los cuales también repercuten en sus subjetividades, aprendizajes y participación dentro de las instituciones educativas,

${ }^{1}$ Este artículo se elabora en base a tesis desarrollada para obtener el grado de Magister en Educación Inclusiva de la Universidad Central de Chile. 
registrándose en un sistema de diferencia en apariencias confirmadas por la evolución del mundo, especialmente por períodos biológicos y cósmicos (Bourdieu, 1998).

\section{Educación inclusiva: Un nuevo desafío para profesores noveles}

Frente al dilema de la diferencia estereotipada y su reproducción en la escuela, es pertinente relevar el rol que juegan los docentes junto con su formación inicial y visibilizar las representaciones que poseen en relación al género. Si bien la formación docente es concebida "como un proceso continuo, sistemático y organizado, que abarca la totalidad de la carrera docente" (Ruffinelli, Cisternas y Córdoba, 2017, p. 17), este estudio se enfocará en el estadio de entrada a la enseñanza (Fuentealba, 2006), período de tiempo que abarca los primeros años de trabajo, en los cuales Marcelo $(1988,2008)$ agrega que los profesores han de realizar la transición desde estudiantes a profesores, identificado como un período de tensiones y aprendizajes, en donde los profesores noveles adquieren más conocimiento profesional. Sumado a esto, los docentes se encuentran en un momento de dualidad, ya que deben preocuparse tanto de enseñar como también de aprender a enseñar (Solís et al., 2016). La decisión de enfocarse en esta etapa del ejercicio docente surge debido a la importancia atribuida a las dificultades que poseen los profesores noveles una vez incorporados al sistema educativo. Estas, descritas por Cornejo (1999, p. 62) siguiendo a Imbernón (1989), tienen relación primero con la falta de capacidad que tiene la formación inicial de preparar a los profesores para la realidad que se vive en las escuelas, principalmente lo que refiere al trabajo en equipo, el clima de aula, la relación con los pares y la adaptación de los contenidos en contextos diversos (Calvo y Camargo, 2015; Ruffinelli, Cisternas y Córdoba, 2017); y segundo, con la misma rutina del quehacer docente (caracterizada como repetitiva y poco solidaria) en que el ejercicio de docentes noveles se desarrolla con un limitado contacto con los pares, enfrentando la problemática del reconocimiento de los otros, quienes influirán y determinarán sus rutinas pedagógicas (Calvo y Camargo, 2015; Romero, Ubillus y Sierra, 2017).

Pero es frente a estas adversidades que se espera que los docentes noveles posean "ideas y habilidades críticas, así como la capacidad de reflexionar, evaluar y aprender sobre su enseñanza de tal forma que mejoren continuamente" (Marcelo, 2008, p. 11). Por tanto, este momento crítico requiere una especial atención en la formación de docentes noveles en la institución educativa a partir de la reflexión sobre la cultura escolar y asumiendo el ingreso al sistema educativo oficial como una posibilidad de nuevos aprendizajes (Calvo y Camargo, 2015; Granados, Tapia y Fernández, 2017).

Esta expectativa se extiende a lo referido sobre la incorporación del enfoque de género que ha logrado permear a la sociedad y transformarse en un tema contingente y cada vez más visible; no obstante, las prácticas reproductivas de las representaciones sociales en torno al género se continúan haciendo presentes, en especial en los espacios educativos. Sumado a que existe escasa o nula presencia del enfoque de género en las diferentes carreras de pedagogía, ilustrativamente, no se hace alusión en los perfiles de egreso ni en la estructura curricular, restringiendo su abordaje en asignaturas asociadas a la inclusión y derechos humanos (Salas y Salas, 2016).

Tal asociación se comprende porque la inclusión escolar ha comenzado a posicionarse hace ya bastantes años como tema prioritario en los planos educativo y social en diversas 
naciones (Echeita y Ainscow, 2011). A pesar de ser un concepto confuso y que en algunos casos se ha entendido como una forma de trabajo con aquellos niños y niñas que poseen necesidades educativas especiales, se ha logrado ampliar este concepto entendiéndolo como un proceso que promueve la superación de aquellos obstáculos que limitan la presencia, la participación y los logros de todas las y los estudiantes (UNESCO, 2017).

A la vista de lo que señala Durán y Giné (2011), que la "formación para la inclusión tiene ante todo como metas ayudar al profesorado a aceptar la responsabilidad del aprendizaje de todos los alumnos, requiriéndose compromiso, afecto, conocimiento de la didáctica, múltiples modelos de enseñanza, reflexión sobre la práctica y trabajo en equipo que promueva el aprendizaje entre los colegas (p. 167). Necesario cuando en Chile "se ha considerado la inclusión escolar como una adaptación relativamente normalizada del currículum, dejando de lado asuntos de tipo interaccional” (Villalobos et al., 2014, p. 173). Esto tiene sentido, cuando la mayor preocupación está concentrada en los resultados de la medición a través del Sistema Nacional de Medición de la Calidad de la Educación. Este énfasis academicista ha restringido la mirada respecto a inclusión.

\section{Representaciones sociales: Aproximaciones a la comprensión en torno al género}

Las representaciones sociales hacen referencia a tipos de conocimiento que juegan un papel sumamente influyente y estereotipado respecto a lo que las personas piensan y en cómo estas organizan su vida cotidiana (Lozano, 2010). Estas configuraciones concuerdan con las de algunos grupos sociales, generando asimismo visiones e interpretaciones colectivas. Es decir, una subjetividad individual y compartida a la vez (Garnique, 2012). Llevándonos a comprender que, si las representaciones de cada individuo no son declaradas, resultan una tarea compleja, pues "la representación social es el paradigma teórico desde el cual deben ser analizadas las relaciones sociales en relación con los procesos de dinámica social y de dinámica psíquica” (Flores, 2007, p. 105).

Al profundizar en esta idea, constatamos que una representación es una especie de articulación de un sistema ideológico (el cual funciona dentro de un procedimiento político) siendo una función normativa de un lenguaje que puede mostrar o distorsionar lo que se considera verdadero o erróneo respecto al género (Butler, 2007). Esto viene a reafirmar que cada sujeto y la "idea" de su propia identidad de género y a la que este posee de otros, no se liga puramente al sexo (hombre-mujer), pues las representaciones como un proceso social son una cuota altamente determinadora de la preferencia estética, ideológica y emocional en cada sujeto, estableciéndose su carácter cambiante e innovador, que deriva de la naturaleza significante de este proceso: la representación como un conjunto de significados nos permiten interpretar lo que nos sucede (Jodelet, 1986).

Si mencionamos el género como uno de los temas en que las representaciones influyen significativamente, podemos destacar que nos referimos a una forma abstracta que es producto de la propia evolución social que ha sufrido el ser humano a lo largo de la historia, por ejemplo, las mujeres como grupo marginado y su evolución en la lucha del reconocimiento por su participación social en términos de derechos humanos. En suma, "el género es una categoría que abarca, efectivamente, lo biológico, pero es, además, una categoría bio-socio-psico-econo-político-cultural” (Lagarde, 1996, p. 3). 
En este sentido, la escuela se resiste a modificar sus prácticas y discursos segmentadores, persistiendo en agrupar en su rutina a las y los estudiantes de acuerdo con el sexo al que pertenecen y "treat gender identity (male/masculine and female/feminine) both as epistemological products and as a binary basis necessary for the operation of a heteronormative logic" (Dornelles y Dal'Igna, 2015, p. 4).

Considerando el panorama, se vuelve pertinente entender que la categoría de género "analiza la síntesis histórica que se da entre lo biológico, lo económico, lo social, lo jurídico, lo político, lo psicológico, lo cultural; implica al sexo, pero no agota ahí sus explicaciones" (Lagarde, 1996, p. 3). Al respecto, Lamas (2000) nos señala que el género debe ser conceptualizado como

el conjunto de ideas, representaciones, prácticas y prescripciones sociales que una cultura desarrolla desde la diferencia anatómica entre mujeres y hombres, para simbolizar y construir socialmente lo que es "propio" de los hombres (lo masculino) y "propio" de las mujeres (lo femenino). (p. 2)

Para efectos de esta investigación, el género se entenderá como una construcción bajo significados e interpretaciones culturales del sexo. En palabras de Butler, "el género no designa a un ser sustantivo, sino a un punto de unión relativo entre conjuntos de relaciones culturales e históricas específicas" (Butler, 2007, p. 61).

Los docentes, al ser parte de la sociedad, incluso inconscientes de ello, van traspasando a sus estudiantes los estereotipos de género no sólo a través de su discurso sino también por medio de sus acciones pedagógicas (Abett, 2014; Flores, 2007; Uribe y Ramírez, 2008), transmitiendo a las y los educandos representaciones que se desenvuelven en el contexto escolar, las cuales llegan a ser homogeneizantes y unificadas, actuando sobre ellos de tal forma que influye de manera evidente en sus comportamientos y creación de sus identidades, llegando a reproducir modelos sobre la base de su formación en la escuela (Díaz y Ruz, 2012).

Considerando que Ortega y Pagés (2018) recomiendan seguir investigando los discursos emergidos de la práctica docente en torno al género, sumado a la reflexión crítica sobre los planes de formación del profesorado, éste artículo tiene por objetivo describir las representaciones que poseen docentes noveles de Educación General Básica respecto a la construcción de género y sus aportes para una Educación Inclusiva, asumiendo que, como participantes de nuevas generaciones de profesionales que han sido formados bajo perspectivas inclusivas, posean representaciones sociales que contemplen perspectivas de género, contribuyendo en su rol a romper con la heteronorma establecida, la cual limitaría a las y los estudiantes en sus trayectorias de vida. Puesto que, por la condición significante de cualquier representación, particularmente la identidad de género como binaria, tanto en sus aspectos figurativo como simbólicos nos permite aludir a lo dinámico y renovador de la misma (Jodelet, 1986).

\section{Método}

La siguiente investigación se enmarca en el paradigma cualitativo-descriptivo, buscando ahondar en las representaciones sociales de docentes noveles con perspectiva de género y cómo estas influyen en el quehacer profesional. Para lograr este objetivo se ha determinado desarrollar un estudio descriptivo, entendiendo que se desarrolla 
considerando aspectos temporales y espaciales particulares; junto a esto se elaboran preguntas enmarcadas en esquemas descriptivos.

Este estudio se desarrollará con una muestra intencionada de cuatro profesores noveles, considerando sus años de ejercicio profesional (de cero a cuatro años) y género (dos hombres y dos mujeres) (cuadro 1).

Cuadro 1. Descripción general de los participantes

\begin{tabular}{|c|c|c|c|c|c|}
\hline AUTORIZA & EDAD & $\begin{array}{l}\text { TÍTULO } \\
\text { PREGRADO }\end{array}$ & $\begin{array}{l}\text { INSTITUCIÓN DE } \\
\text { PREGRADO }\end{array}$ & $\begin{array}{l}\text { INSTITUCIÓN } \\
\text { LABORAL }\end{array}$ & $\begin{array}{l}\text { AÑOS DE } \\
\text { DOCENCIA }\end{array}$ \\
\hline Daniela & 30 & $\begin{array}{l}\text { Profesora } \\
\text { Educación General } \\
\text { Básica }\end{array}$ & $\begin{array}{l}\text { Universidad } \\
\text { Metropolitana de } \\
\text { Ciencias de la } \\
\text { Educación }\end{array}$ & $\begin{array}{l}\text { Liceo Dr. Juan } \\
\text { Verdaguer } \\
\text { Planas }\end{array}$ & 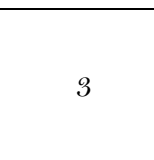 \\
\hline $\begin{array}{l}\text { María } \\
\text { Fernanda }\end{array}$ & 24 & $\begin{array}{l}\text { Profesora de } \\
\text { Educación General } \\
\text { Básica }\end{array}$ & $\begin{array}{l}\text { Universidad } \\
\text { Central de Chile }\end{array}$ & $\begin{array}{l}\text { Centro } \\
\text { Educacional } \\
\text { Matías Cousiño }\end{array}$ & 2 \\
\hline César & 26 & $\begin{array}{l}\text { Profesor de } \\
\text { Educación } \\
\text { General Básica }\end{array}$ & $\begin{array}{l}\text { Universidad } \\
\text { Central de Chile }\end{array}$ & $\begin{array}{l}\text { Liceo José } \\
\text { Victorino } \\
\text { Lastarria }\end{array}$ & 2 \\
\hline Rubén & 28 & $\begin{array}{l}\text { Licenciado en } \\
\text { Historia Profesor } \\
\text { de Educación } \\
\text { General Básica }\end{array}$ & $\begin{array}{l}\text { Universidad } \\
\text { Arturo Prat } \\
\text { Universidad } \\
\text { Miguel de } \\
\text { Cervantes }\end{array}$ & $\begin{array}{l}\text { Colegio San } \\
\text { Martín de } \\
\text { Porres }\end{array}$ & 3 \\
\hline
\end{tabular}

Fuente: Elaboración propia.

Se utilizará como instrumento de recolección de información la entrevista semiestructurada. Este tipo de entrevista permite mayor flexibilidad ya que las preguntas planteadas tienen la posibilidad de acomodarse a las y los entrevistados; y también permiten "revelar el conocimiento existente de manera que se pueda expresar en forma de respuestas y, por tanto, hacerse accesible a la interpretación” (Flick, 2007).

La técnica de análisis de los datos será el análisis del discurso, el cual es una herramienta sofisticada de análisis en las ciencias sociales que permite relacionar el discurso con aquellas condiciones objetivas y subjetivas de producción, circulación y consumo de los mensajes. Dentro de sus principales características, este dispositivo puede contener aspectos relativos a los modelos mentales, a la identidad, los roles, los géneros discursivos, las ideologías y las relaciones de dominación presentes en los participantes (Sayago, 2014). $\mathrm{El}$ análisis del discurso nace de la emergencia de un mensaje común, en donde el texto o relato tiene sentido porque hay un contexto.

Para la validación del análisis a realizar, se aplicará lo que Denzin (1989) llamó la triangulación de la teoría, la cual se caracteriza por reconocer que las interpretaciones dadas por dos investigadores nunca serán de forma idéntica, lo que permitiría comparar sus datos para así lograr la validación (citado en Stake, 1998, p. 98).

\section{Resultados}

Los hallazgos se ordenaron en las categorías que se exponen en el cuadro 3. 
Cuadro 3. Categorías de análisis

\begin{tabular}{ll}
\hline Concepciones de género & Influencia familiar \\
\hline Estereotipos de género & $\begin{array}{l}\text { Educación no sexista } \\
\text { Sociedad conservadora/reproductora }\end{array}$ \\
\hline Situaciones de menoscabo & $\begin{array}{l}\text { Rol reproductor de la escuela } \\
\text { Practicas escolares }\end{array}$ \\
Formación inicial con enfoque de género & Rol del docente \\
\hline Afectividad & Acciones que promueven una Educación \\
\hline Modificaciones curriculares & Inclusiva con perspectiva de género. \\
Afirmación de la idea de diferencia &
\end{tabular}

Fuente: Elaboración propia.

\subsection{Representaciones de género en docentes noveles: transición conceptual y persistencia dicotómica}

Las representaciones que emergen desde los discursos de los docentes noveles, vinculadas a la conceptualización del término "género", contienen relevantes inconsistencias y contradicciones en todos entrevistados de esta investigación. Esta categoría es vinculada de manera inmediata a atributos biológicos dicotómicos existentes: pene - hombre / vagina - mujer. Por tanto, se visibilizan elementos que dan cuenta de una perspectiva unida al sexo, entendido como lo biológico, lo cual podría estar influenciado por el contexto histórico-social al que han estado sometidas sus representaciones, lo que se reafirma en reflexiones de De Beauvoir (1981), Subirats (1994), Bourdieu (1998) y Butler (2007).

Sin embargo, y pese a las declaraciones expuestas, emergen matices en el discurso que contribuyen a una mutación de la significación asociada a esta categoría, por la presencia de subjetividades, ideas y creencias que se anteponen a una condición sexual como un factor determinante del género. Lo que se ilustra en el discurso de María Fernanda:

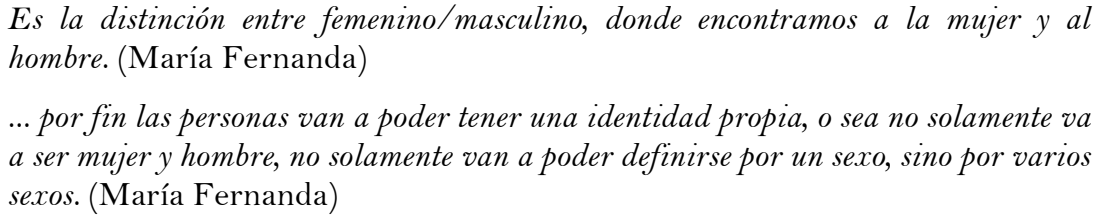

La transición conceptual en análisis se puede atribuir a un cambio paradigmático como consecuencia de las recientes manifestaciones sociales que han permitido la visibilización, con el objetivo de llegar a un reconocimiento de las diferentes manifestaciones de género existentes, situación que ha sensibilizado a las nuevas generaciones, las cuales posibilitan esta transición, derribando perspectivas conservadoras, estereotipadas y fijas. Este tránsito enriquecería las prácticas pedagógicas, pues demuestra que los docentes noveles contemplan y reconocen la existencia de una diversidad de géneros y sexualidades que hasta hoy se han visto invisibilizados en las escuelas. Sobre todo, dan cuenta de un pensamiento en construcción inconcluso que posibilita un dialogo y la construcción de un “otro” lenguaje, una “otra” práctica.

Si bien podemos destacar una transición en relación con el concepto de género, no podemos negar los estereotipos que poseen y que operan como limitantes de márgenes sociales en la asignación de espacios, en especial en aquellos que hacen referencia a la rasgos y valoraciones asignadas a cada género heteronormado. En este sentido, la persistencia dicotómica y los roles de padre y madre obstaculizan un mayor tránsito a posicionamientos más abiertos y plurales: 
En cambio, el hombre lo vemos asociado más al que trae el sustento económico, la persona que gana mucho más que la mujer porque es un "líder". En cuanto a roles el hombre no está tan preocupado de la crianza como la mujer, la mujer totalmente tiene el rol de ser madre. (María Fernanda)

A las niñas se les enseña a ser metódicas, ordenadas, paso a paso, porque esta construcción matriarcal hace que las niñas actúen con delicadeza, con cuidado, poniendo atención, mientras que el niño está preocupado de hacer desorden o es más laxo en el tema de poder establecer un método de trabajo: si es desordenado no importa, de todas formas, es niño y va a jugar a la pelota. (Rubén)

Estos estereotipos de género se entienden con carácter de obligatoriedad para los miembros de grupos sociales y, de acuerdo con esto, limitarían a todo estudiante en sus sueños y experiencias ya que las desigualdades y prejuicios de género, tal como señalan los participantes, son causa de que niñas y niños vivan en una especie de sociedad segmentada, con expectativas independientes y ampliamente divergentes entre sí (Díaz y Ruz, 2012). Dentro de los discursos de los participantes se observa que la escuela clasifica a las y los estudiantes, permitiendo que "ellos" puedan tener libertades y caer en el error, mientras que a "ellas" se les sugiere el rigor y tributo a lo privado, pues el estereotipo asignado determina que el sexo femenino es delicado y disciplinado; observándose que la mirada inclusiva es muy restringida, puesto que no se estarían eliminando las barreras para resguardar una auténtica participación en todos los ámbitos de la existencia humana, barreras que podrían derrumbarse cuando la cotidianeidad laboral y personal les interpela:

En ninguna situación me he encontrado menoscabado, rechazado o vulnerado hasta hoy, donde nace este nuevo concepto del feminismo, porque sólo por el hecho de ser hombre soy agresor y no por ser hombre puedo ser victima o sentir parte de lo que están sintiendo ellas. (César)

En lo laboral sí, a veces pienso y siento que en una institución donde trabajan muchas mujeres y las jefaturas son todas femeninas siento que muchas veces los papeles de representación lo ocupan hombres. Por ejemplo, tiene que haber un profesor representante de los profes, y es un hombre. Cosas como esa me violentan un poco, no tanto, pero lo hacen. (Daniela)

Con los hombres que estudian para enseñanza básica y para párvulo también hay discriminación: no se les da mucho trabajo, se piensa que un hombre en relación con niños pequeños puede generar más casos de abuso, siendo que también hay casos de mujeres que han abusado de niños. Creo que socialmente el cuidado es un rol que se le ha dado a la mujer. (Daniela)

En síntesis, las representaciones de los docentes noveles han revelado que estos habitan en diversos relatos y recurren a unos y otros para analizar su experiencia. Sin embargo, siguen vinculando el concepto de género al de sexo, pues se deduce que no existe una preparación respecto a las diferencias que implican ambos términos. Asimismo, demuestran una persistencia dicotómica, admitiendo la existencia de los estereotipos dentro de sus discursos al caracterizar mediante ejemplos cómo hombres y mujeres son educados en la familia y cómo esto se reproduce dentro de las dinámicas de la escuela, atribuyendo espacios y roles que participan en la construcción de la personalidad. En suma, se observa en sus discursos dificultades para superar el enfoque androcéntrico y la incoherencia entre los discursos inclusivos y de enfoque de género. 


\title{
4.2. Facilitadores y barreras para una Educación Inclusiva en y desde el enfoque de género: promoción de la afectividad y negación a la diferencia
}

\section{La afectividad como facilitador}

Dentro de las declaraciones, emergen posibles facilitadores que contribuirían a una educación con perspectiva de género en el marco de una Educación Inclusiva. Si bien sólo un entrevistado destaca a la afectividad como un facilitador, creemos que este hallazgo podría ser un elemento esencial para el desarrollo de una Educación Inclusiva. César lo plantea a lo largo de su relato:

\begin{abstract}
La primera y yo creo que la más importante que falta demasiado, es un análisis socioafectivo del sujeto, yo soy fiel partidario de que el sujeto que entra a la formación inicial docente debiese ser evaluado cognitiva y socioafectivamente, qué es lo que entiende, porque él va a transmitir no solamente conocimientos, sino una visión del mundo. Si esa visión del mundo es errática y estática, es muy "cuadrada” por decirlo de una manera, está muy difícil que sea capaz de aceptar, de reconocer, de valorar al otro que es su estudiante. (César)
\end{abstract}

La universidad debiera considerar la educación para las emociones, más psicología, talleres complementarios (...). Potenciar el tema afectivo, el cómo ayudar a que ese estudiante se encuentre, se reconozca y se valore. (César)

Le falta esa parte, al currículum hoy día, entender que para tener un sujeto integro hay que contemplar sus habilidades conceptuales y afectivas. (César)

$\mathrm{El}$ entrevistado, al establecer que son las casas de estudio quienes deben evaluar bajo ciertos criterios a sus estudiantes para ingresar a su formación inicial, afirma que los docentes en formación deberían cumplir con características afectivas previas para poder iniciarse en la docencia. De esta manera, las instituciones asegurarían obtener profesionales que logren dar cuenta de los múltiples sentimientos, emociones y pasiones que son parte de una manifestación propia del ser humano y vinculante a dimensiones cognitivas del mismo que pretenden dar cuenta de su visión del mundo; comprendiendo visión del mundo como el conjunto de elementos cognitivos, prácticas socioculturales y dimensiones morales, éticas y políticas que poseen los individuos, permitiendo una evaluación de la realidad tal como lo señala Bortolan (2017).

La evaluación de la afectividad, según señala en primera instancia, debiese ser un requisito previo al ingreso a la formación inicial docente. Una idea compleja de materializar sin que se pierda la densidad de este fenómeno, que da cuenta del grado de afectación en y desde los sucesos vitales vinculados a la concepción y promoción de género que impacta en los docentes y sus aspectos cognitivos, como lo son las creencias y juicios de cada sujeto, sumados a los deseos y acciones de los mismos. En este sentido, la propuesta de César es relevante ya que la afectividad cumpliría una función estructurante del contexto, de la realidad y la concepción actual del mundo en múltiples términos, entre ellos el género, dando a entender que desde y en la afectividad las concepciones dicotómicas, fijas y jerarquizables se pueden superar. En otras palabras, reconocer la vulnerabilidad del ser humano permitiría la construcción de "otra" forma de habitar el mundo.

Por otro lado, al contemplar la afectividad como un elemento del currículum, el entrevistado la está reconociendo como indispensable en los procesos de enseñanzaaprendizaje posibilitando una perspectiva de género. Pues abrirse a la experiencia del otro y consentir que me afecte, posibilitará abandonar estereotipos, representaciones que afectan o reducen al otro. De esta forma, estos procesos afectivos predicen un tipo de aprendizaje más integral y efectivo porque se basan en una estructura motivacional en las 
y los estudiantes, apostar por la coeducación afectivosexual como espacio educativo, socializador, imprescindible para construir una cultura inclusiva en el marco de la educación para la ciudadanía democrática y los derechos humanos (Escobar, 2015; Venegas, 2017).

Negación a la diferencia como barrera para la Educación Inclusiva

En los discursos de los y las participantes se aprecia una inconsistencia con el término "diferencia”, la que podría generarse por constituirse como concepto polisémico con alta carga ideológica en su definición al utilizarse en narrativas contrarias entre sí. Discursos que versan que todos tenemos las mismas habilidades en primera instancia, para luego indicar que todos somos diferentes.

\begin{abstract}
No establezco diferenciación de género, no está planteado desde ese punto de vista, no planifico ni construyo material pensando conscientemente en que no establezco diferenciación de género, es porque simplemente yo no lo tengo registrado como algo que deba hacer, me pasa, al contrario: tengo naturalizado que no existe diferenciación de género". (Rubén)Es que todos somos iguales, todos tenemos las mismas habilidades y que ninguno quede en desventaja, que todos tengamos las mismas herramientas, ya sea porque tengamos una diferencia sexual diferente o una discapacidad pero que todos pertenezcamos a los mismos porque en realidad todos venimos de lo mismo. (María Fernanda)

Yo creo que desde ahí se parte todo, si nosotros tenemos una Educación Inclusiva desde los inicios de nuestra edad, se va a poder enseñar también que las personas todas somos diferentes en el aspecto de capacidades, preferencia sexual, todo. Una Educación Inclusiva debe contemplar un enfoque de género. (María Fernanda)
\end{abstract}

Concepciones que dan cuenta de las ideas que rodean al concepto de democracia y justicia social que se tensiona en "combinar la igualdad fundamental de todos sus miembros con las «desigualdades justas» que surgen de una competencia meritocrática equitativa” (Dubet, 2012, p. 43). En otras palabras, igualar a los estudiantes significa omitir aquellas características individuales que determinan sus procesos de aprendizaje (acceso a la información, evaluación diferenciada e incluso modificaciones curriculares). Se cae en el error de homogeneizar, volviendo a la cuestión específica de la idea de las personas con discapacidad, exigiendo y ofreciendo servicios especializados para "los diferentes", a grupos particulares de niños, los cuales son difíciles de satisfacer en aulas ordinarias, desviándonos del objetivo que persigue una Educación Inclusiva (Echeita y Ainscow, 2011). Identificar las diferencias en función de las particularidades de los y las sujetos, promueve una pedagogía de las diferencias desde la cual el otro y la Otra es considerada como legítima Otra.

\title{
4.3. Aportes para una Educación Inclusiva: performatividad del lenguaje y de las acciones de docentes noveles
}

A partir de las representaciones extraídas de los discursos de docentes, se pueden apreciar dos elementos claves para el desarrollo de una Educación Inclusiva: el lenguaje y las acciones. Entendiendo performatividad como un mecanismo mediante el cual a través de la reiteración de los discursos se produce lo que se nombra (Butler, 2002). Butler nos señala que de la misma forma en que el lenguaje y las acciones han creado nuestras subjetividades (y con esto nuestras concepciones de género), también pueden modificarse y subvertir las concepciones de nuestros cuerpos, las cuales se han encontrado arraigadas por cientos de años a modelos conservadores, binarios, heteronormados y patriarcales. Lo anterior es descrito por Rubén planteando: 


\begin{abstract}
El lenguaje es súper importante (...). Entonces cómo se construye el lenguaje a partir de las necesidades es muy importante para ir destrabando esto. Se necesita un lenguaje inclusivo, y no me refiero al todos, bajo ningún punto de vista. El lenguaje es súper simple y preciso a la vez: todos y todas, alumnos y alumnas. Si quiero hablar en plural: estudiantes. El lenguaje te da las herramientas para que todo el mundo sea incluido en las distintas actividades de la vida. El lenguaje es primordial a la hora de establecer estos cambios. (Rubén)
\end{abstract}

En este sentido, estaríamos frente a una gran oportunidad para potenciar la Educación Inclusiva con perspectiva de género. Así lo destaca Rubén al señalar al lenguaje como una herramienta transformadora. Si bien él es el único entrevistado que alude de manera explícita al uso del lenguaje, se ha logrado observar que en las declaraciones de los otros y otras entrevistadas las modificaciones en el discurso demuestran un cambio de perspectiva (transición en concepciones de la categoría de género), por lo tanto, cambios paradigmáticos en las generaciones noveles de los y las docentes.

Se vuelven relevantes los alcances que tienen en común los docentes noveles al reconocer y proponer modificaciones de las prácticas escolares que, de ser transformadas, contribuirían en buena parte a una Educación Inclusiva con perspectiva de género. Destacan la propuesta de una modificación de alto impacto al currículum y los programas de estudio, pues de esta manera se podrían modificar las prácticas que, hasta ahora, se encuentran masculinizadas, por ejemplo, los recursos educativos bibliográficos, desconociendo la presencia de la mujer y otros géneros en la configuración histórica de la humanidad. Así, en las prácticas pedagógicas, deben reconocer las individualidades de cada estudiante, modificando espacios y rutinas escolares en función de garantizar la participación de los diversos géneros, no sólo de los y las estudiantes, también en los y las gestores de las instituciones educativas, cargos públicos y administrativos.

\title{
5. Conclusiones
}

Contemplando los hallazgos se observa que una relación dilemática entre igualdad vs diferencia está presente en los discursos de docentes noveles, infiriendo que las instituciones de Formación Inicial Docente aún no logran construir una narrativa de la pedagogía de las diferencias, preocupante, ya que una vez en el ejercicio laboral es posible que se vean tentados a habitar añejas y persistentes narrativas normalizadoras. Asimismo, actualmente la discusión de políticas nacionales no logra -o no desea- profundizar en este aspecto, manteniendo un discurso basado en la igualdad normativa, permitiendo la permanencia y profundización del paradigma de la igualdad de oportunidades que limita el avance hacia el desarrollo de una igualdad sustantiva y la igualdad de posiciones, en la que no sólo se asegure el ingreso a una educación de calidad e inclusiva, sino a una justicia social.

Si bien la igualdad normativa ha sido una herramienta exitosa en el aumento de la matrícula de estudiantes en el sistema escolar regular, generaría una restricción del desarrollo personal en cada sujeto, ya que esta se encarga de homogeneizar promoviendo discursos meritocráticos, no reconociendo aquellas características individuales que determinan los procesos de aprendizaje de cada estudiante.

Los y las docentes, al hablar de los cambios necesarios para una Educación Inclusiva con perspectiva de género, principalmente se dirigen al cambio en políticas públicas, 
priorizando la modificación en las estructuras macro (currículum, leyes, puestos de mando, etc.) por sobre las micro, subordinando las prácticas pedagógicas a estas estructuras.

Considerando lo anterior, el rol transformador social del docente se somete a los diversos dispositivos del sistema educativo, por lo tanto ¿cuánto de lo que desarrollan los y las docentes lo hacen por convicción propia o por un simple mandato superior? Plantear la necesidad de generar dispositivos "vigilantes" o "reeducadores" es limitar la pedagogía a seguir una lista de normativas y leyes, desplazando la responsabilidad institucional (tanto universitarias como escolares) de generar docentes críticos y de potenciar aquellas cualidades indispensables en el ejercicio de la docencia, más aún cuando se habla de Educación Inclusiva con perspectiva de género.

Respecto al objetivo que orientó nuestra investigación es importante resaltar que la concepción de Educación Inclusiva es restringida, a pesar de que existen en la actualidad múltiples discursos que apelan a no limitarla a la condición de discapacidad y no fundamentarla desde la perspectiva del déficit. Los hallazgos nos vienen a demostrar que su significado aún no está resuelto en las negociaciones, de las cuales todos participamos, faltando además la discusión en torno a la igualdad de oportunidad versus la igualdad de posiciones, en este caso entre hombres y mujeres y otras identificaciones. Es decir, aun en el marco de la Educación Inclusiva no se logra construir un discurso que acoja identificaciones asociadas al género.

Una de las limitaciones de esta investigación es la ausencia de análisis de los programas de formación inicial docente; esta herramienta habría aportado a dar mayor densidad al estudio. No obstante, a partir de los discursos obtenidos en esta microinvestigación, surgen posibles líneas de investigación, proponiendo en primera instancia indagar respecto a cuál es la incidencia de las relaciones familiares en las trayectorias profesionales y específicamente en apropiarse de una perspectiva de género.

\section{Referencias}

Abett, P. (2014). Educación y género: Una reflexión sobre los principios de igualdad y diversidad en la educación chilena en el escenario de la reforma educativa actual. Revista de Pedagogía Crítica Paulo Freire, 16, 35-47.

Althusser, L. (1988). Ideología y aparatos ideológicos del estado; Freud y Lacan. Recuperado de https://perio.unlp.edu.ar/teorias2/textos/m3/althusser.pdf

Bonino, L. (2003). Masculinidad hegemónica e identidad masculina. Dossiers Feministes, 6, 7-36.

Bortolan, A. (2017). Affectivity and moral experience: An extended phenomenological account. Phenomenology and the Cognitive Sciencies, 16, 471-490. https://doi.org/10.1007/s11097016-9468-9

Bourdieu, P. (1998). La dominación masculina, traducción de Joaquín Jordá. Barcelona: Anagrama.

Butler, J. (2002). Cuerpos que importan: Sobre los limites materiales y discursivos del sexo. Barcelona: Paidós.

Butler, J. (2007). El género en disputa: El feminismo y la subversión de la identidad. Barcelona: Paidós.

Cabello, C. (2018). Educación no sexista y binarismos de género. Agitaciones feministas y disidencias sexuales secundarias en la escuela. En F. Zerán (Ed.), Mayo feminista. La rebelión contra el patriarcado (pp. 21-34). Santiago de Chile: LOM ediciones. 
Calvo, G. y Camargo, M. (2015). Hacer escuela en la formación de docentes noveles. Páginas de Educación, 8(1), 73-91.

Ceplak, M. M. (2013). Heteronormativity: School, ideology, and politics. Journal of Pedagogy, 4(2), 162-187. https://doi.org/10.2478/jped-2013-0009

Cornejo Abarca, J. (1999). Profesores que se inician en la docencia: Algunas reflexiones al respecto desde América Latina. Revista Iberoamericana De Educación, 19, 51-100. https://doi.org/https://doi.org/10.35362/rie1901055

De Beauvoir, S. (1981). El segundo sexo. Buenos Aires: Editorial Siglo XX.

Dornelles, P. y Dal'Igna, M. (2015). Gender, sexuality and age: Heteronormativity in pedagogical practices of physical education in schools. Educação e Pesquisa, 41, 1585-1599. https://doi.org/10.1590/S1517-9702201508142508

Dubet, F. (2012). Los límites de la igualdad de oportunidades. Nueva Sociedad, 239, 42-56.

Durán, D. y Giné, C. (2011). La formación del profesorado para la educación inclusiva: Un proceso de desarrollo profesional y de mejora de los centros para atender la diversidad. Revista Latinoamericana de Educación Inclusiva, 5(2), 153-170.

Echeita, G. y Ainscow, M. (2011). La educación inclusiva como derecho. Marco de referencia y pautas de acción para el desarrollo de una revolución pendiente. Tejuelo: Didáctica de la lengua y la Literatura, 12, 26-46.

Escobar, M. (2015). Influencia de la interacción alumno-docente en el proceso enseñanzaaprendizaje. Paakat: Revista de Tecnología y Sociedad, 5(8), art 4.

Flick, U. (2007). Introducción a la investigación cualitativa. Madrid. Editorial Morata. Paideia.

Flores, R. (2007). Representaciones de género de profesores y profesoras de matemática y su incidencia en los resultados académicos de alumnos y alumnas. Revista Iberoamericana de Educación, 43, 103-118.

Fuentealba, R. (2006). Desarrollo profesional docente: Un marco comprensivo para la iniciación pedagógica de los profesores principiantes. Foro Educacional, 10, 65-106.

Garnique, C. (2012). Las representaciones sociales: Los docentes de educación básica frente a la inclusión escolar. Perfiles Educativos, 34(137), 99-118.

https://doi.org/10.22201/iisue.24486167e.2012.137.34119

Granados, J., Tapia, A. y Fernández, J. (2017). La construcción de la identidad de los docentes noveles: Un análisis desde las teorías apriorísticas. Revista de Docencia Universitaria, 15(2), 163-178. https://doi.org/10.4995/redu.2017.6746

Imbernón, F. (1989). La formación inicial y la formación permanente del profesorado. Dos etapas de un mismo proceso. Revista Interuniversitaria de Formación del Profesorado, 6, 487-499.

Jodelet, D. (1986). La representación social: Fenómenos, concepto y teoría. Barcelona: Paidós.

Lagarde, M. (1996). La multidimensionalidad de la categoría género y del feminismo. Metodología para los estudios de género. Ciudad de México: Instituto de Investigaciones Económicas.

Lamas, M. (2000). Diferencias de sexo, género y diferencia sexual. Cuicuilco, 7(18), 1-22.

Lozano, R. L. E. (2010). Representaciones sociales sobre identidad de género, en docentes en formación como licenciados en educación básica. Entre Comillas, 13, 62-80.

Marcelo, C. (1988). Profesores principiantes y programas de inducción a la práctica docente. Enseñanza ङ゚ Teaching: Revista Interuniversitaria de Didáctica, 6, 61-80.

Marcelo, C. (2008). El profesorado principiante: Inserción a la docencia. Barcelona: Octaedro. 
Ortega, D. y Pagès Blanch, J. (2018). Género y formación del profesorado: Análisis de las guías docentes del área de didáctica de las ciencias sociales. Contextos Educativos. Revista de Educación, 21, 53-66. https://doi.org/10.18172/con.3315

Ruffinelli, A., Cisternas, T. y Córdoba, C. (2017). Iniciarse en la docencia: Relatos de once experiencias. Santiago de Chile: Ediciones Universidad Alberto Hurtado.

Salas, N. y Salas, M. (2016). Tiza de colores: Hacia la enseñanza de la inclusión sobre diversidad sexual en la formación inicial docente. Revista Latinoamericana de Educación Inclusiva, 10(2), 73-91. https://doi.org/10.4067/S0718-73782016000200006

Sayago, S. (2014). El análisis del discurso como técnica de investigación cualitativa y cuantitativa en las ciencias sociales. Cinta de Moebio, 49, 1-10. https://doi.org/10.4067/So7 17-554X2014000100001

Solís, M., Núñez, C., Contreras, I. y Ritterhaussen, S. (2016). Inserción profesional docente: Problemas y éxitos de los profesores principiantes. Estudios Pedagógicos, 42(2) 331-342. https://doi.org/10.4067/So7 18-07052016000200019

Stake, R. E. (1998). Investigación con estudio de casos. Madrid: Morata.

Strear, M. (2017). Forecasting an inclusive future: School counseling strategies to deconstruct educational heteronormativity. Professional School Counseling, 20(1), 47-56. https://doi.org/10.5330/1096-2409-20.1a.47

Subirats, M. (1994). Panorámica sobre la situación educativa de las mujeres: Análisis y políticas. Pensar las diferencias. Barcelona: Universitat de Barcelona.

UNESCO. (2017). Guía para asegurar la inclusión y la equidad en la educación. París: UNESCO.

Venegas, M. (2017). Coeducar las relaciones afectivo-sexuales para promover la igualdad sexual y de género y la justicia social. Revista Internacional de Educación para la Justicia Social, 6(2), 13-28. https://doi.org/10.15366/riejs2017.6.2.001

Villalobos, B., Carrasco, C., López, V., Ramírez, M. y Morales, M. (2014). Inclusión y violencia: Prevalencia de victimización entre pares en estudiantes que participan en programas de integración escolar. Revista Latinoamericana de Educación Inclusiva, 8(2), 161-178.

Zerán, F. (2018). Mayo feminista. La rebelión contra el patriarcado. Santiago de Chile: LOM.

\section{Breve CV de las autoras}

\section{Violeta Quiroz Fuentes}

Profesora de Enseñanza Básica con Mención en Historia, Geografía y Ciencias Sociales. Magister en Educación Inclusiva Universidad Central. Postítulo en Psicología Educacional Universidad de Chile. Licenciada en Educación Universidad Metropolitana de Ciencias de la Educación. Actualmente se desempeña como docente de aula en el establecimiento The Little School en Santiago de Chile. Ha profundizado sus estudios en Derechos Humanos y Estudios de Género. ORCID ID: https://orcid.org/OOOO-OOO18238-8413. Email: quiroz.violeta@gmail.com

\section{Melissa Sennas Vásquez}

Profesora de Enseñanza Básica con Mención en Lenguaje y Comunicación. Magister en Educación Inclusiva y Licenciada en Educación de la Universidad Central de Chile. Actualmente se desempeña como docente de aula en el establecimiento Cristóbal Colón, 
Melipilla, Chile. Se ha especializado en la aplicación de estrategias de enseñanzaaprendizaje en los primeros niveles de enseñanza básica. ORCID ID:

https://orcid.org/0000-0003-0334-4602.Email: melissa.sennas@gmail.com

\section{Sylvia Contreras Salinas}

Doctora en pedagogía de la diversidad sociocultural por la Universidad Complutense de Madrid, Magister en Educación con Mención en Currículo y Evaluación. Socióloga y Profesora de Educación Diferencial. Ha trabajado como docente e investigadora en diversas universidades chilenas. Actualmente se desempeña en el Departamento de Educación de la Universidad de Santiago de Chile. Su línea de investigación intersecta las temáticas de género, saberes, migración, infancia y educación. ORCID ID:

https://orcid.org/0000-0003-2297-2399.Email: sylvia.contreras.s@usach.cl 